\title{
Avaliação e correlação de parâmetros fisiológicos e índices bioclimáticos de vacas holandês em diferentes estações.
}

Evaluation and correlation of physiological parameters and bioclimatic indexes holstein cows in different seasons.

\author{
André Sanches de Avila', langlio Márcio Travassos Duarte Jácome², Andressa Faccenda', \\ Dejani Maira Panazzolo ${ }^{3}$ Éderson Rogério Müller ${ }^{4}$
}

\author{
'Mestrando em Produção e Nutrição Animal - Universidade Estadual do Oeste do Paraná (UNIOESTE) \\ ${ }^{2}$ Docente do Curso de Zootecnia - Universidade Federal de Santa Maria (UFSM) \\ ${ }^{3}$ Mestranda em Produção Animal - Universidade Federal do Rio Grande do Sul (UFRGS) \\ ${ }^{4}$ Graduando em Zootecnia - Universidade Federal de Santa Maria (UFSM)
}

\begin{abstract}
Resumo
Objetivou-se com este trabalho determinar os parâmetros fisiológicos de vacas da raça Holandês em lactação durante a estação de inverno e de primavera e correlacionar tais parâmetros com os índices bioclimáticos. Foram utilizadas nove vacas da raça Holandês em um delineamento inteiramente ao acaso, em arranjo de parcelas subdivididas com medidas repetidas no tempo. Os dados foram coletados durante o inverno e a primavera, nos quais foram medidas a frequência respiratória (FR), a frequência cardíaca (FC), a temperatura superficial (TS) e a temperatura retal (TR) dos animais, além da temperatura do ar, a umidade relativa e a temperatura de globo negro. Foram calculados o Índice de Temperatura e Umidade (ITU), Índice de Temperatura de Globo Negro e Umidade (ITGU) e Carga Térmica Radiante (CTR). O ITU, ITGU e CTR apresentaram aumentos no período da tarde, sugerindo um ambiente passível de estresse. Não foram observadas diferenças estatísticas $(\mathrm{P}>0,05)$ entre as estações do inverno e da primavera para nenhuma das variáveis fisiológicas. Somente a FC mostrou-se acima dos limites fisiológicos para bovinos. A FC, FR e TS apresentaram correlação positiva e significativa $(\mathrm{P}<0,01)$ com o ITU e o ITGU e a CTR. Contudo, não foi detectado estresse térmico nos animais na estação de inverno e da primavera, embora os parâmetros fisiológicos tenham apresentado correlação com os índices bioclimáticos.
\end{abstract}

Palavras Chave: ITU, ITGU, CTR, vacas leiteiras, produção de leite

\begin{abstract}
The objective of this study was to determine the physiological parameters of Holstein cows in milking during the winter and spring season and correlate these parameters with the bioclimatic indexes. We used nine Holstein cows in a completely randomized delimitation in a subdivided plot arrangement with repeated measures in time. Data were collected during the winter and spring, which were measured respiratory rate (RR), heart rate (HR), superficial temperature (ST) and rectal temperature (RT) from animals, besides the air temperature, the relative humidity and black globe temperature. It was calculated the temperature and humidity index (THI), the Black Globe Temperature and Humidity Index (BGTH) and Radiant Heat Load (RHL). The THI, BGTH and RHL showed increases in the afternoon suggesting an environment susceptible to stress. There were no statistical differences $(\mathrm{P}>0.05)$ between the seasons of winter and spring for any of the physiological variables. Only HR was above physiological limits for cattle. HR, RR and ST showed significant positive correlation $(\mathrm{P}<0.01)$ with the THI and BGTH and RHL. However, heat stress was not detected in animals in the winter and spring seasons, although the physiological parameters have shown a correlation with the bioclimatic indexes.
\end{abstract}

Keywords: THI, BGHT, RHL, dairy cows, milk productivity 


\section{INTRODUÇÃO}

O Rio Grande do Sul apresenta-se como um grande produtor de leite e seu nível de competitividade o situa entre os mais eficientes produtores do Brasil. A cadeia leiteira responde por mais de $7 \%$ do PIB do agronegócio do estado, o que auxilia no seu desenvolvimento econômico (FINAMORE et al., 2009). Devido a essa grande importância da atividade, cada vez mais se busca produzir com qualidade e minimizar estresses que possivelmente venham a causar diminuição na produção animal.

O clima é um conjunto de fenômenos meteorológicos particulares de cada região que interferem no metabolismo dos animais. Condições adversas como o frio ou calor excessivo podem gerar estresse térmico, pois o organismo necessita alterar sua fisiologia para manter a sua homeostase.

O estado do Rio Grande do Sul está localizado segundo Buriol, Estefanel e Chagas (2006) pela classificação climática de Koeppen em uma região de clima temperado úmido (Cfa) caracterizada por temperatura média do ar no mês mais quente superior ou igual a $22^{\circ} \mathrm{C}$ e ocorrência de precipitação em todos os meses do ano. A presença de chuvas em todas as estações pode provocar alta umidade do ar prejudicando o conforto térmico dos animais em temperaturas acima da zona termoneutra do animal. West (2003) destaca que a dissipação de calor do animal para o ambiente é prejudicada com a alta umidade, pois o gradiente térmico é reduzido.

$\mathrm{O}$ ambiente em que os animais estão submetidos pode ser classificado pelos índices bioclimáticos, que tem por objetivo expressar o conforto que o animal possui em determinadas condições. Isso depende de fatores como umidade relativa, intensidade do vento, precipitações, radiação térmica e pelas superfícies de contato. Os resultados desses índices possibilitam uma avaliação mais precisa da situação do ambiente e a comparação de resultados zootécnicos com animais de diferentes regiões (FERREIRA, 2005).

O estresse térmico pode também ser estudado pela mensuração de variáveis como a temperatura interna do animal, temperatura superficial, frequência respiratória e cardíaca, pois segundo Perissinoto et al. (2006), os indicadores fisiológicos e comportamentais são uma forma de quantificar esse estresse.

Objetivou-se neste trabalho avaliar os parâmetros fisiológicos de vacas da raça Holandês em lactação durante a estação de inverno e da primavera e correlacionar tais parâmetros com os índices bioclimáticos.

\section{MATERIAL E MÉTODOS}

O experimento foi realizado em uma propriedade rural da cidade de Palmeira das Missões, localizada no noroeste do Rio Grande do Sul, a $639 \mathrm{~m}$ de altitude, entre as coordenadas de latitude sul $27^{\circ} 53^{\prime} 55^{\prime \prime}$ e longitude oeste $53^{\circ} 18^{\prime}$ 9". O experimento ocorreu no período de julho de 2010 até setembro de 2010.

Foram utilizadas nove vacas da raça Holandês preta e branca em fase de lactação. Foi adotado um delineamento inteiramente ao acaso, em arranjo de parcelas subdivididas com medidas repetidas no tempo, sendo as parcelas constituídas pelas estações (inverno e primavera) e as subparcelas constituídas pelas três coletas em cada estação. Os animais tinham acesso à pastagem de aveia consorciada com azevém com sal mineral e água a vontade, recebiam suplementação no cocho de concentrado com $20 \%$ de PB e silagem de milho. $\mathrm{O}$ manejo dos animais iniciava-se com a primeira ordenha às 6 horas, após os animais seguiam para os canzis onde recebiam $50 \%$ da oferta diária de concentrado, em seguida eram conduzidos à pastagem. Às 11 horas recebiam silagem de milho no cocho onde permaneciam até a ordenha da tarde. A segunda ordenha era realizada às 17 horas, e após, novamente seguiam aos canzis onde recebiam o restante de concentrado.

Os dados foram coletados durante duas estações (inverno e primavera) com medidas repetidas no tempo. As coletas foram realizadas às 6 horas e às 17 horas, nas quais foram medidas a frequência respiratória, a frequência cardíaca, a temperatura superficial e a retal dos animais. Também foram coletadas a temperatura do ar, a umidade relativa do ar na sala de ordenha no horário entre as 7 horas e 17 horas e a temperatura de globo negro em intervalos de duas horas.

A frequência respiratória e a frequência cardíaca foram mensuradas pela contagem dos movimentos do flanco e pelas batidas do coração, respectivamente, durante 20 segundos e o valor foi multiplicado por três para se obter a frequência dos movimentos respiratórios e dos batimentos cardíacos por minuto. Para aferição da temperatura retal, utilizou-se um termômetro digital, inserido aproximadamente $5 \mathrm{~cm}$ no reto. A temperatura superficial foi obtida pela média da temperatura corporal coletada com um termômetro de infravermelho em quatro pontos do animal, sendo dois pontos aferidos em local de pelagem preta e dois pontos em local de pelagem branca.

A mensuração da temperatura ambiente e da umidade relativa do ar foram coletadas por um 
datalogger termohigrômetro digital com mensurações a cada 30 minutos. Para as medidas de temperatura de globo negro, foi utilizado termômetro de mercúrio inserido em esfera de polietileno preta.

O Índice de Temperatura e Umidade (ITU) da sala de ordenha foi calculado a partir da fórmula de Thom (1958) descrita a seguir:

$$
\mathrm{ITU}=\mathrm{ta}+0,36 \mathrm{t}_{\mathrm{po}}+41,5
$$

Onde:

ta: temperatura do ar $\left({ }^{\circ} \mathrm{C}\right)$;

$\mathbf{t}_{\text {po: }}$ temperatura de ponto de orvalho $\left({ }^{\circ} \mathrm{C}\right)$

Oo: cálculo do Índice de Temperatura de Globo Negro e Umidade (ITGU) foi realizado de acordo com a fórmula proposta por Buffington et al. (1981)

$$
\text { ITGU }=\operatorname{tgn}+0,36 t_{\mathrm{po}}+41,5
$$

Em que:

tgn: temperatura do globo negro, $\left({ }^{\circ} \mathrm{C}\right)$;

$\mathbf{t}_{\mathrm{po}}$ : temperatura de ponto de orvalho $\left({ }^{\circ} \mathrm{C}\right)$.

O Índice de Carga Térmica Radiante (CTR)

foi calculado como proposto por Esmay (1979):
$\mathrm{C} \mathrm{T} \mathrm{R}=$
$\mathrm{t}$
$(\mathrm{T} \mathrm{M} \mathrm{R})^{4}$

$\mathrm{TMR}=100((2.51(\mathrm{vv}) 05(\mathrm{tg}-\mathrm{ta})+(\operatorname{tg} / 100) 4) 0.25)$

Sendo:

TRM: Temperatura Média Radiante;

vv : velocidade do vento $(\mathrm{m} / \mathrm{s})$;

$\operatorname{tg}$ : temperatura de globo negro $\left({ }^{\circ} \mathrm{C}\right)$;

ta : temperatura ambiente $\left({ }^{\circ} \mathrm{C}\right)$;

t : 5.67 * $10-8 \mathrm{~K}-4 . \mathrm{W} / \mathrm{m}^{2}$ (Constante de

Stefan-Boltzmann).

Os parâmetros fisiológicos dos animais foram submetidos à análise de variância e teste de Tukey a $5 \%$ de probabilidade. Posteriormente, foi realizada uma correlação simples de Pearson entre os parâmetros fisiológicos dos animais e os índices bioclimáticos, utilizando o Sistema de Análises Estatísticas e Genéticas programa (SAEG), desenvolvido pela Universidade Federal de Viçosa (UFV, 1997).

\section{RESULTADOS E DISCUSSÃO}

Na figura 1, encontram-se os valores de temperatura, umidade, Índice de Temperatura e Umidade, Índice de temperatura de Globo negro e Umidade e Carga Térmica Radiante do período da manhã e da tarde nos dias de coleta.

A zona termoneutra para um determinado animal foi definida por Nããs (1989) como uma faixa limitada pelas temperaturas máximas e mínimas consideradas ótimas para a produção do mesmo, nas quais a manutenção da homeotermia ocorre com uma mobilização mínima dos mecanismos responsáveis pela termorregulação. A mesma autora ainda se refere à faixa de 4 a $24^{\circ} \mathrm{C}$ como confortável para vacas em lactação. As temperaturas observadas neste estudo durante o período da tarde ultrapassaram a zona termoneutra dessa categoria animal até mesmo durante a estação de inverno. A umidade relativa do ar manteve-se acima de $53 \%$ durante todo o experimento, sendo que os valores mais elevados ocorreram no período da manhã independente da estação.

O ITU, ITGU e CTR apresentaram aumentos no período da tarde em consequência ao aumento da temperatura. Houve também uma pequena elevação desses índices na primavera em relação ao inverno, refletindo a alteração climática

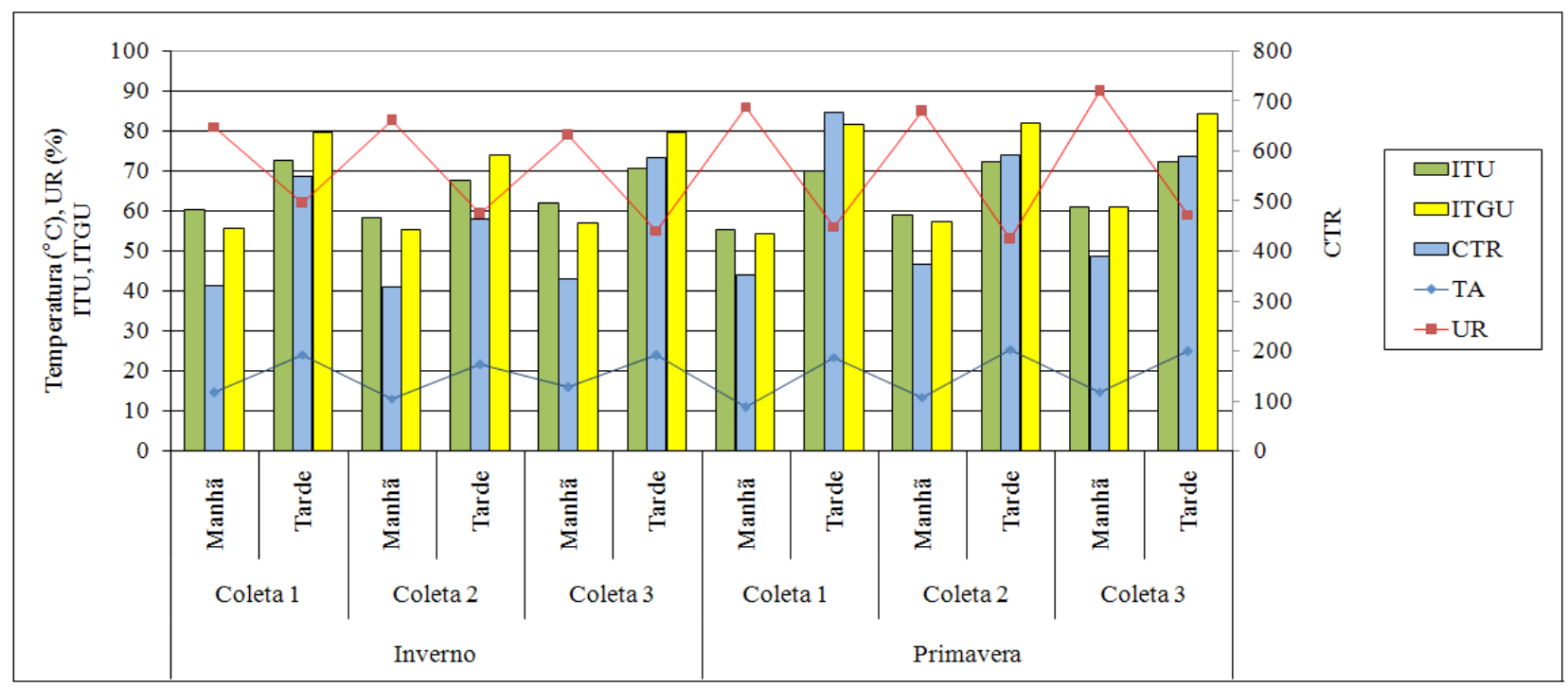

Figura 1 - Valores de temperatura ambiente, umidade relativa, Índice de Temperatura e Umidade, Índice de Temperatura de Globo Negro e Umidade e Carga Térmica Radiante nas diferentes coletas de inverno e da primavera 
em função da mudança de estação.

Armstrong (1994) classificou o estresse térmico em ameno quando o ITU estiver na faixa de 72 a 78. Nesse estudo, os valores de ITU apresentaram-se acima de 72 durante a tarde em uma coleta do inverno e duas coletas na estação da primavera. Em relação ao ITGU, Baêta (1985) destaca que valores na faixa de 74 a 78 caracterizam situação de alerta e valores de 79 a 84 caracterizam que os animais encontram-se em situação de perigo podendo acarretar baixo rendimento. Desse modo, durante as tardes de inverno, o ITGU indicou situação entre alerta e perigo, enquanto que durante a primavera indicou situação de perigo ao conforto térmico dos animais, chegando a valores de até 84,5.

A CTR está intimamente relacionada às trocas térmicas por radiação entre o animal e o ambiente, com isso em regiões tropicais são desejáveis os menores valores possíveis de CTR (SILVA, 2000). Nesse trabalho, esse índice foi maior na estação da primavera em relação ao inverno, refletindo a maior temperatura ambiente e maior radiação solar nessa estação.

$\mathrm{Na}$ tabela 1, estão descritos os valores médios dos parâmetros fisiológicos referentes ao período da manhã para as duas estações avalia- das. Não foram observadas diferenças estatísticas $(\mathrm{P}>0,05)$ entre as estações do inverno e da primavera para nenhuma das variáveis fisiológicas avaliadas. A temperatura retal e a frequência cardíaca também não diferiram $(\mathrm{P}>0,05)$ entre os períodos de coleta em nenhuma das estações. A temperatura superficial da manhã diferiu $(\mathrm{P}<0,05)$ entre as coletas nas duas estações, enquanto a frequência respiratória diferiu $(\mathrm{P}<0,05)$ entre as coletas da primavera. Tal fato pode ter ocorrido pelo aumento da temperatura média ambiente ao longo das estações o que resultou no aumento das variáveis fisiológicas dos animais.

A temperatura retal da manhã manteve-se dentro dos valores fisiológicos que segundo Martello et al. (2004) para bovinos adultos situa-se entre 37,5 a $39,3^{\circ} \mathrm{C}$. A frequência respiratória e a frequência cardíaca também mantiveram-se dentro do ideal em ambas as estações, pois de acordo com Hahn e Mader (1997) quando a frequência respiratória for de 60 movimentos por minuto os animais não apresentam estresse térmico ou esse está em nível baixo.

o. Quanto à frequência cardíaca, Rossarolla (2007) destaca que para bovinos adultos os valores fisiológicos situam-se entre 60 e 70 batimentos por minuto.

Tabela 1 - Valores médios de temperatura retal, temperatura superficial, frequência respiratória e frequência cardíaca do período da manhã de vacas Holandês em lactação

\begin{tabular}{|c|c|c|c|}
\hline \multicolumn{4}{|c|}{ Temperatura Retal $\left({ }^{\circ} \mathrm{C}\right)$} \\
\hline Estação & $1^{\circ}$ coleta & $2^{\circ}$ coleta & $3^{\circ}$ coleta \\
\hline Inverno & $37,7 \mathrm{aA}$ & $37,8 \mathrm{aA}$ & $37,7 \mathrm{aA}$ \\
\hline Primavera & $37,4 \mathrm{aA}$ & $37,7 \mathrm{aA}$ & $37,8 \mathrm{aA}$ \\
\hline \multicolumn{4}{|c|}{ Temperatura Superficial $\left({ }^{\circ} \mathrm{C}\right)$} \\
\hline Estação & $1^{\circ}$ coleta & $2^{\circ}$ coleta & $3^{\circ}$ coleta \\
\hline Inverno & $21,6 \mathrm{aA}$ & $25,1 \mathrm{bA}$ & $26,6 \mathrm{cA}$ \\
\hline Primavera & $25 \mathrm{aA}$ & $27,1 \mathrm{bA}$ & $28,7 \mathrm{cA}$ \\
\hline \multicolumn{4}{|c|}{ Frequência Respiratória (mov.min ${ }^{-1}$ ) } \\
\hline Estação & $1^{\circ}$ coleta & $2^{\circ}$ coleta & $3^{\circ}$ coleta \\
\hline Inverno & $24 \mathrm{aA}$ & $24,8 \mathrm{aA}$ & $26,3 \mathrm{aA}$ \\
\hline Primavera & $18,9 \mathrm{aA}$ & $21 \mathrm{abA}$ & $22,9 \mathrm{bA}$ \\
\hline \multicolumn{4}{|c|}{ Frequência Cardíaca (bat.min ${ }^{-1}$ ) } \\
\hline Estação & $1^{\circ}$ coleta & $2^{\circ}$ coleta & $3^{\circ}$ coleta \\
\hline Inverno & 60,6 aA & $66 \mathrm{aA}$ & $71,1 \mathrm{aA}$ \\
\hline Primavera & $63 \mathrm{aA}$ & $63,4 \mathrm{aA}$ & $61,7 \mathrm{aA}$ \\
\hline
\end{tabular}

Médias seguidas por letras minúsculas diferentes na linha diferem estatisticamente pelo teste de Tukey a 5\% de probabilidade. Médias seguidas por letras maiúsculas diferentes na coluna diferem estatisticamente pelo teste de Tukey a 5\% de probabilidade. 
$\mathrm{Na}$ tabela 2, estão descritos os valores médios dos parâmetros fisiológicos referentes ao período da tarde para as duas estações avaliadas. As variáveis fisiológicas no período da tarde tiveram um comportamento semelhante ao período da manhã, não diferindo $(\mathrm{P}>0,05)$ entre as estações avaliadas. A frequência cardíaca e a temperatura retal não foram diferentes entre as coletas $(\mathrm{P}>0,05)$, a temperatura superficial da primavera e a frequência respiratória do inverno diferiram $(\mathrm{P}<0,05)$ nos períodos das coletas aumentando no decorrer da estação. Navarini et al. (2009), encontraram diferenças na temperatura superficial de bovinos sendo que esta foi $0,9^{\circ} \mathrm{C}$ superior nos animais que permaneceram ao sol em relação aos que tinham acesso à sombra.

A temperatura retal e a frequência respiratória da tarde mantiveram-se dentro dos valores fisiológicos. Ferreira et al. (2006) em seus trabalhos constataram que a TR no período da manhã apresentou-se dentro da faixa de normalidade para bovinos tanto no verão quanto no inverno, porém à tarde, os valores de TR foram superiores aos limites fisiológicos, evidenciando que os animais não conseguiram manter a homeotermia.

As frequências cardíacas observadas nas duas estações foram superiores aos valores consi- derados fisiológicos, pois ultrapassaram os 70 bat. $\mathrm{min}^{-1}$, chegando a atingir 81 bat.min ${ }^{-1}$. No entanto, apenas a alteração dessa variável não é suficiente para determinar a ocorrência de estresse térmico nos animais, visto que além da temperatura, outros fatores como raça, idade, trabalho muscular, ingestão de grande quantidade de alimento e ruminação (ROSSAROLLA, 2007) podem influenciar esse parâmetro.

A FC apresentou correlação positiva e significativa $(\mathrm{P}<0,01)$ com o ITU e o ITGU e correlação também positiva e significativa $(\mathrm{P}<0,05)$ com a CTR. No que se refere à temperatura superficial e a frequência respiratória, ambas demonstraram correlações positivas e altamente significativas $(\mathrm{P}<0,01)$ com todos os índices bioclimáticos que utilizam como base para seus cálculos a temperatura ambiente. Medeiros e Vieira (1997) destacam que quando a temperatura ambiente está acima do limite crítico também há o aquecimento da pele e essa sensação de calor é conduzida ao hipotálamo. Este comanda uma dilatação dos vasos sanguíneos superficiais, o que acarreta maior quantidade do sangue junto à superfície do corpo para ser resfriado, passando por condução para a pele e daí para o ambiente, por radiação. Os mesmos autores ainda destacam que a aceleração do ritmo

Tabela 2 - Valores médios de temperatura retal, temperatura superficial, frequência respiratória e frequência cardíaca do período da tarde de vacas Holandês em lactação

\begin{tabular}{|c|c|c|c|}
\hline \multicolumn{4}{|c|}{ Temperatura Retal $\left({ }^{\circ} \mathrm{C}\right)$} \\
\hline Estação & $1^{\circ}$ coleta & $2^{\circ}$ coleta & $3^{\circ}$ coleta \\
\hline Inverno & $38,5 \mathrm{aA}$ & $38,5 \mathrm{aA}$ & $38,5 \mathrm{aA}$ \\
\hline Primavera & $38,2 \mathrm{aA}$ & $38,3 \mathrm{aA}$ & $38,4 \mathrm{aA}$ \\
\hline \multicolumn{4}{|c|}{ Temperatura Superficial $\left({ }^{\circ} \mathrm{C}\right)$} \\
\hline Estação & $1^{\circ}$ coleta & $2^{\circ}$ coleta & $3^{\circ}$ coleta \\
\hline Inverno & $30,7 \mathrm{aA}$ & $29,8 \mathrm{aA}$ & $30,6 \mathrm{aA}$ \\
\hline Primavera & $31,1 \mathrm{aA}$ & $30,8 \mathrm{aA}$ & $33 \mathrm{bA}$ \\
\hline \multicolumn{4}{|c|}{ Frequência Respiratória (mov.min ${ }^{-1}$ ) } \\
\hline Estação & $1^{\circ}$ coleta & $2^{\circ}$ coleta & $3^{\circ}$ coleta \\
\hline Inverno & 35,3 aA & $39,4 \mathrm{abA}$ & $46,9 \mathrm{bA}$ \\
\hline Primavera & $33,8 \mathrm{aA}$ & $31,9 \mathrm{aA}$ & $42,4 \mathrm{aA}$ \\
\hline \multicolumn{4}{|c|}{ Frequência Cardíaca (bat.min (1) $^{-1}$} \\
\hline Estação & $1^{\circ}$ coleta & $2^{\circ}$ coleta & $3^{\circ}$ coleta \\
\hline Inverno & $81 \mathrm{aA}$ & $80,3 \mathrm{aA}$ & $79,9 \mathrm{aA}$ \\
\hline Primavera & $71,6 \mathrm{aA}$ & $73,1 \mathrm{aA}$ & $77,3 \mathrm{aA}$ \\
\hline
\end{tabular}

Médias seguidas por letras minúsculas diferentes na linha diferem estatisticamente pelo teste de Tukey a 5\% de probabilidade. Médias seguidas por letras maiúsculas diferentes na coluna diferem estatisticamente pelo teste de Tukey a $5 \%$ de probabilidade. 
Tabela 3 - Coeficientes de correlação de Pearson entre os parâmetros fisiológicos e os índices bioclimáticos

$\begin{array}{cccc}\text { Variáveis } & \text { ITU } & \text { ITGU } & \text { CTR } \\ \text { Frequência Cardíaca } & 0,8463 & 0,8123 & 0,6964 \\ \text { Frequência Respiratória } & 0,8611 & 0,857 & 0,7668 \\ \text { Temperatura Superficial } & 0,8488 & 0,8985 & 0,8626 \\ \text { Temperatura Retal } & -0,2376 & -0,2239 & -0,2145\end{array}$

respiratório também ocorre devido ao aumento da temperatura, e tem como função gerar perda de calor por via evaporativa, pois o aquecimento do ar inspirado rouba calor do organismo no interior do aparelho respiratório.

A TR apresentou correlação negativa, porém não significativa $(\mathrm{P}>0,05)$ em relação aos índices bioclimáticos avaliados. Tal fato demonstra que a temperatura retal não acompanhou o aumento dos índices bioclimáticos durante o dia. Gaughan et al. (2000) ressaltam que o aumento dessa variável não é considerado como forma de termorregulação, mas como o resultado do equilíbrio térmico, ou seja, é a consequência incapacidade dos mecanismos de termorregulação em eliminar o excesso de calor, desse modo como não ocorreu estresse considerável nos animais esse parâmetro não foi significativamente alterado.

\section{CONCLUSÕES}

Não foi detectado estresse térmico nos animais na estação de inverno e da primavera.

A frequência cardíaca, frequência respiratória e a temperatura superficial apresentaram correlação positiva e significativa com o ITU, ITGU e a CTR.

Novos estudos são necessários para estimar os níveis críticos dos índices bioclimáticos para a região noroeste do Rio Grande do Sul, visto que os índices avaliados apontaram para um ambiente com condições climáticas estressantes aos animais, o que não ocorreu ao se avaliar os parâmetros fisiológicos dos mesmos.

\section{REFERÊNCIAS BIBLIOGRÁFICAS}

ARMSTRONG, D. V. Heat stress interaction with shade and cooling. Journal of Dairy Science, Champaign, v.77, p.2044-2050, 1994.

BAÊTA, F.C. Responses of lactating dairy cows to the combined effects of temperature, humidity and wind velocity in the warm season. 1985. $218 \mathrm{f}$. The- sis (PhD.) University of Missouri, Columbia, 1985.

BUFFINGTON, D.E. et al . Black globe humidity index (BGHI) as comfort equation for dairy cows. Transaction of the ASAE, St. Joseph, v.24, n.3, p.711-14, 1981.

BURIOL, G. A.; ESTEFANEL, V.; CHAGAS, A. C. A vegetação do estado do Rio Grande do Sul em função dos modelos fitoclimáticos de Martone e Thornthwaite \& Hare e da classificação climática de Koeppen. In:CONGRESSO BRASILEIRO DE METEOROLOGIA, 14., 2006, Florianópolis. Anais eletrônicos... Florianópolis: CBMET, 2006. Disponível em: <http://www.cbmet.com/cbmfiles/149f73a79359e1e2104088 e5da6770c168.pdf>. Acesso em : 3 nov. 2011.

ESMAY, M.L. Principles of animal environment. Westport: AVI, 1978. 358 p. (Environmental engineering in agriculture and food series)

FERREIRA, F. et al. Parâmetros fisiológicos de bovinos cruzados submetidos ao estresse calórico. Arquivo Brasileiro de Medicina Veterinária e Zootecnia, Belo Horizonte, v.58, n.5, out. 2006.

FERREIRA, R.A. Maior produção com melhor ambiente: para aves, suínos e bovinos. Viçosa: Aprenda Fácil, 2005.

FINAMORE, E.B.M.C. et al. Características dos produtores de leite do RS: uma análise a partir do Corede Nordeste. In: CONGRESSO DA SOCIEDADE BRASILEIRA DE ECONOMIA, ADMINISTRAÇÃO E SOCIOLOGIA RURAL, 47., 2009, Porto Alegre. Anais... Porto Alegre: Sociedade Brasileira de Economia, Administração e Sociologia Rural, 2009.

GAUGHAN, J. B. et al. Respiration Rate - Is It a Good Measure of Heat Stress in Cattle? Asian-Australasian Journal of Animal Sciences, v.13, p.329332, Jul. 2000.

HAHN, G.L.; MADER, T.L. Heat waves in relation 
on thermoregulation, feeding behavior, and mortality of feedlol cattle. In : International Livestock 42 Environment Symposium, 5., Mennesota, 1997. Proceedings. ST. Joseph: ASAE,1997. p.125-129.

MARTELLO, L. S. et al. Respostas fisiológicas e produtivas de vacas holandesas em lactação submetidas a diferentes ambientes. Revista Brasileira de Zootecnia, Viçosa, v.33, n.1, Viçosa, jan./fev. 2004.

MEDEIROS, L. F. D.; VIEIRA, D. H. Bioclimatologia animal. 1997. Disponível em: http://www. iz.ufrj.br/zootecnia_draa/biblioteca/Fernando/ apostila\%20I.pdf. Acesso em: 12 nov. 2013.

NÃ̃̃S, I.A. Princípios de conforto térmico na produção animal. São Paulo: Ícone, 1989.

NAVARINI, F. C. et al. Conforto térmico de bovinos da raça nelore a pasto sob diferentes condições de sombreamento e a pleno sol. Engenharia Agrícola, Jaboticabal v.29, n.4, p.508-517, out./dez. 2009.

PERISSINOTTO, M. et al. Efeito da utilização de sistemas de climatização nos parâmetros fisiológicos do gado leiteiro, Engenharia Agrícola, Jaboticabal, v.26, n.3, p.663-671, set./dez. 2006.

ROSSAROLLA, G. Comportamento de vacas leiteiras da raça holandesa, em pastagem de milheto com e sem sombra. 2007. Dissertação (Mestrado) - Santa Maria, 2007.

SILVA, R. G. Introdução a Bioclimatologia Animal. São Paulo:Nobel, 2000. 450p.

THOM, E.C. The discomfort index. Weatherwise, Washington, v.12, p.57-9, 1959.

UNIVERSIDADE FEDERAL DE VIÇOSA - UFV. SAEG- Sistema para análises estatísticas e genéticas. Versão 7.1. Viçosa, MG: 1997. 150p (Manual do usuário).

WEST, J. W. Effects of heat-stress on production in dairy cattle. Journal of Dairy Science, Champaign, v. 86, p. 2131-2144, 2003. 